\title{
Garfield number: on some characteristics of Eugene Garfield's first and second order co-authorship networks
}

\author{
Wolfgang Glänzel $^{1,2} \cdot$ Mehmet Ali Abdulhayoğlu $^{2}$
}

Received: 5 November 2017/Published online: 14 December 2017

(C) Akadémiai Kiadó, Budapest, Hungary 2017

\begin{abstract}
In this note we give an overview of the first- and second-order collaboration network reflected by Eugene Garfield's publications in scientific journals. Although he had only a quite limited number of co-authors and co-publications, his co-authors' own collaboration networks generate a large world-wide and multidisciplinary coverage. The classical model of co-authorship network is the Erdös network with the Erdös Number indicating the shortest co-authorship path through which an author is connected with Paul Erdös. The two networks, generated by Erdős and Garfield, respectively, show completely different patterns and characteristics but illustrate the ways how ideas of great scholars and pioneers disseminate and influence the respective scientific communities.
\end{abstract}

Keywords Eugene Garfield · Erdős Number · Nework analysis · Co-authorship analysis · Citation impact

\section{Introduction}

Eugene Garfield ${ }^{1}$ was one of the most prominent and influential information scientists and practitioners for a half-century. The emergence and rise of scientometrics during the last decades would just be inconceivable without his pioneering work. He has founded one of the first and most significant scientific information-service and research centres in the world, the ISI in Philadelphia (USA), and was the Inventor of Web of Science. He created several bibliographic indexes and databases that formed and still form the fundament of professional scientometricians' daily work. Without his ingenious innovation of citation

\footnotetext{
${ }_{1}^{1}$ Part of the introduction is based on two recent pieces by the author (Braun et al. 2017; Glänzel 2017).
}

Wolfgang Glänzel

wolfgang.glanzel@kuleuven.be

1 ECOOM and Department of MSI, KU Leuven, Louvain, Belgium

2 ECOOM, KU Leuven, Louvain, Belgium 
indexing, the field of present-day scientometrics, as we know it, would not be imaginable. His name is also associated with many important ideas and concepts, among other with writing citation historiography and mapping the world of science. And he was an indefatigable scientific writer. His industrious creative activity brought forth an oeuvre that embraces thousands of commentaries, pieces, articles, chapters and books. Garfield was also an important collaborator in research, although the number of his co-authored publications was rather moderate. He initially worked most notably with his team but collaborated later with several scientists from other institutes, partially in longer-term co-author relationship, but with most of his co-authors only occasionally. By the interdisciplinary nature of the field of information science the collaboration network, created by him, his co-authors (1st order relationship) and their own collaborators (2nd order relationship), covers practically most areas of the sciences, social sciences and humanities. This makes the role of Eugene Garfield in the universe of scientific knowledge creation truly unique. In what follows, we will study this universe in terms of collaboration, researcher connectivity and subject coverage. The complexity of the underlying structures does only allow kind of snapshots shedding some light on the entirety. We will proceed from a very prominent scenario that has inspired us to designing this study. The huge collaboration network generated by Paul Erdös and his co-authors covering the complete mathematics and related fields is maybe the most known and analysed productivity and collaboration structure in scientific research (cf. Erdős Number Project 2017). There might be parallels and analogies between the universes of Erdös and Garfield, however, there are some important factors resulting from the two scientists personalities, their research topics and the role in their community and the society that makes the two worlds completely different and almost incommensurable. This is all the more a reason to study the exciting world of Garfields publication and collaboration network while having first a look at the prototype of all researcher connectivity models.

\section{The Erdős network and Erdős number}

Paul Erdős (1913-1996) was one of the most influential mathematicians of the twentieth century. He was famous for his enormous productivity and his openness for co-operation. This specific type of co-authorship, which is also manifested by his particular scholarly work, is an intrinsic value of scholarly communication in mathematics. In mathematics, problems are addressed by a scholar and then often solved by colleagues, but not necessarily together. This, in mathematics, may already imply co-authorship, let alone if working on solutions to the mathematical problems those are found jointly. On the other hand, mathematics is traditionally a domain of single-authorship. Because of his enormous output accompanied by an extraordinary and field-unspecific co-operativity the idea of creating the Erdös number emerged. It is not quite clear, who has actually proposed this basically "simple" network measure first. It appeared in the mathematics literature in 1969, in an article by Goffman (1969). The number is nothing else but the shortest path connecting an author with Erdös in the complete co-author network created by Paul Erdös and his $n$-th order co-authors, which is iteratively generated by always adding the coauthors of co-authors, who are not already members of the network. According to this process, Erdős himself has, by definition, Erdős number 0, his immediate co-authors have number 1, the co-authors of authors with Erdős number 1 have number 2 unless they are not direct co-authors of Erdős, and so on. The distribution of authors by finite Erdös numbers reveals interesting network properties. The data are based on MathSciNet, the electronic version of the Mathematical Reviews database of the American Mathematical 
Society and provided by The Erdös Number Project ${ }^{2}$ of Oakland University. A total of nearly 270,000 out of 401,000, that is, two-thirds of all different authors in the database have a finite Erdös number the maximum of which amounts to 13. The distribution is almost symmetric with a median of 5. The average Erdös number is 4.65 (see Fig. 1).

Like the h-index in the Web of Science Core Collection and Elsevier Scopus database is used to express publication productivity and impact by one number, the Erdös number is used as a proxy for researchers connectivity and prominence. Unlike most bibliometric measures, such as, for instance the h-index, a lower Erdős number expresses closeness to Erdös and is associated with higher connectivity. In the meantime there are several modifications and supplements to the Erdös number (just as for the h-index in bibliometrics) allowing for adjusting for the number of joint publications and network related indicators (cf. The Erdős Number Project 2017) have been developed.

As already mentioned above, mathematics is a domain of single-authorship. According to the Project data, $62.4 \%$ of documents indexed in the database are single-authored, $27.4 \%$ have two co-authors, $8.0 \%$ three co-authors, $1.7 \%$ are by four co-authors, $0.5 \%$ by more than five authors. This applied to all documents in the database, no distinction is made for the publication year. Surprisingly enough, this is very much in line with the situation in mathematics in the early 1980s according to Clarivate Analytics Science Citation Index Expanded (cf. Glänzel 2002).

This is, in a nutshell, the specific situation in mathematics. The patterns found in coauthorship networks here may certainly not be generalised or even transferred to another field. The differences between productivity and collaboration patterns and the subjectspecific effects of co-operativity on scientific productivity in several fields have already been studied by Braun et al. (2001) and Glänzel (2002).

Here we have to stop for a moment. The above example applies to mathematics papers. Mathematicians might also have published in journals and proceedings outside the scope of Mathematical Reviews with more and different co-authors. Opening the universe to a multidisciplinary environment, including all peculiarities of inter- and cross-disciplinary research, would result in networks that might resist any systematic study when using an equivalent to the Erdös number. The number of authors with Erdös number greater than 2 is already mushrooming by two-three orders of magnitude, its pendent in an interdisciplinary field such as information science, which entertains relationships with practically all fields of the sciences, social sciences and humanities, would however grow out of any scope and perspective. On the other hand, while the Erdős model remains in a relatively well-delineated research field, an analogous model applied to an interdisciplinary context would shed light of interesting cognitive structures that might be an interesting challenge worth to be met. Eugene Garfield plaid a central role in our field so that, inspired by the Erdős model, we conducted a network analysis for authors with Garfield numbers 1 and 2 . The main challenge to us was to map the dissemination of knowledge through co-authorship and to uncover the cognitive structures underlying this network. All data have carefully been cleaned manually.

\section{The Garfield network}

In the following experiment, Eugene Garfield is considered the central node in the authorship network based on all documents indexed in Clarivate Analytics Web of Science Core Collection (WoS). We define the Garfield number analogously to the Erdös number.

\footnotetext{
${ }^{2}$ Retrieved from the project website at http://www.oakland.edu/enp/trivia/.
} 


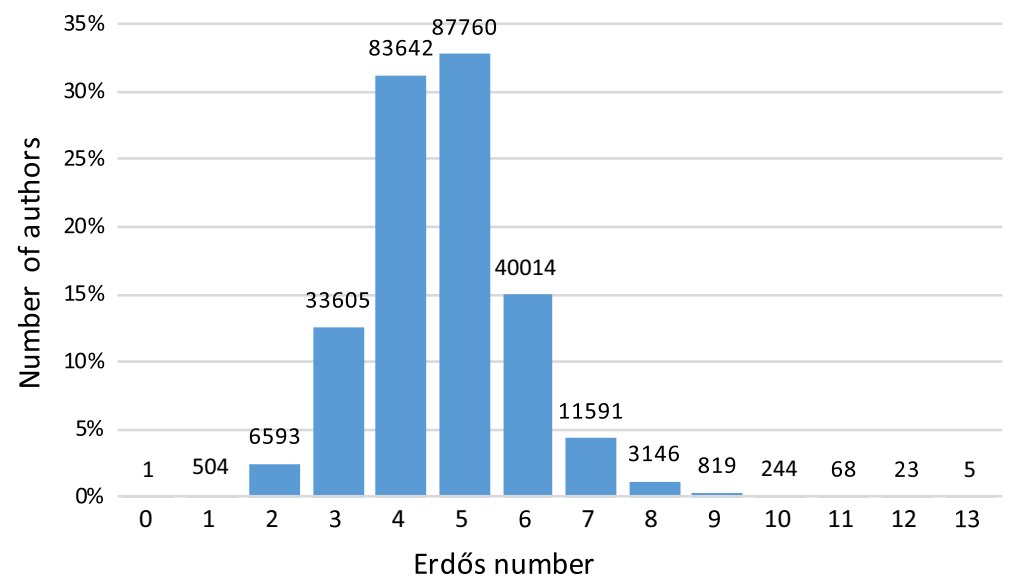

Fig. 1 Distribution of authors by finite Erdős numbers (labels give the absolute numbers). Data sourced from "The Erdős Number Project"

We have considered all document types but we have discarded seven publications (one correction, four discussions and two letters), associated with membership in consortia, which, in our opinion, does not express true co-authorship. Eugene Garfield was a very productive author with 1531 scientific documents indexed in the Web of Science Core Collection (WoS) since 1955. The first striking observation is that the lion's share of these documents (about 96\%) are single-authored. Below we give the distribution of the singleauthored papers according to their document types (Table 1). Eugene Garfield had 51 different co-authors, including colleagues from ISI as well as prominent researchers from different institutes, countries and research fields. A list of their number arranged by document type is given in Table 2. The distribution of co-authors by document type largely reflects that of his single-authored papers, except the large number of Editorial Material

Table 1 Number of documents by Eugene Garfield in the WoS database by document type. Data sourced from Clarivate Analytics Web of Science Core Collection

\begin{tabular}{lrc}
\hline Document type & Number & Share $(\%)$ \\
\hline Article & 778 & 52.96 \\
Editorial material & 564 & 38.39 \\
Letter & 67 & 4.56 \\
Meeting abstract & 12 & 0.82 \\
Item about an individual & 9 & 0.61 \\
Correction & 8 & 0.54 \\
Biographical-item & 7 & 0.48 \\
Article; proceedings paper & 6 & 0.41 \\
Review & 5 & 0.34 \\
Proceedings paper & 4 & 0.27 \\
Correction, addition & 3 & 0.20 \\
Reprint & 3 & 0.20 \\
Note & 2 & 0.14 \\
Book review & 1 & 0.07 \\
Total & 1469 & 100.00 \\
\hline
\end{tabular}


Table 2 Number of Eugene Garfield's different co-authors according to the WoS database by document type. Data sourced from Clarivate Analytics Web of Science Core Collection

\begin{tabular}{lcc}
\hline Document type & Number & Share $(\%)$ \\
\hline Article & 29 & 46.77 \\
Article; proceedings paper & 6 & 9.68 \\
Biographical-item & 1 & 1.61 \\
Book review & 2 & 3.23 \\
Editorial material & 4 & 6.45 \\
Letter & 7 & 11.29 \\
Meeting abstract & 6 & 9.68 \\
Note & 1 & 1.61 \\
Proceedings paper & 2 & 3.23 \\
Reprint & 4 & 6.45 \\
Total & 62 & 100.00 \\
\hline
\end{tabular}

which were written and published by Garfield alone. Almost $90 \%$ of these papers were published in his Current Contents and The Scientist. We have nevertheless decided to include these document types for two reasons. First, they have attracted more than 2500 citations and a small part of those were co-authored and have thus contributed to the network that we wish to study. From the 62 co-authored articles published with 51 different co-authors, we have identified the individual co-authors and their own collaboration network. Finally, we have obtained a small network consisting of the 51 first-order authors in the network ("Garfield number 1") and the 1350 larger one of second-order authors ("Garfield number 2"). The following subsections will be devoted to the empirical analysis of the two networks.

\section{The first-order network (Garfield number $=1$ )}

The first order network is formed by Eugene Garfield's co-authorship links; it is a simple ego-centric network, no matter if we include links between all vertices or not. Like in the case of the Erdös network, only the shortest path matters. The evolution of the number of Garfield's immediate co-authors is given in Table 3. The share of his co-authored papers somewhat increased in the last two decades but this has to do with the decreasing number of publication, which, in turn, is a consequence of structural changes in the publication output. In particular, till 1993 Garfield published numerous papers in Current Comments. These publications are mostly commentaries and essayistic work. Traditional research papers formed the minority of his work. Therefore, the share of co-authored papers is

Table 3 Evolution of Garfield's co-authored papers in the mirror of the Web of Science. Data sourced from Clarivate Analytics Web of Science Core Collection

\begin{tabular}{lcrrr}
\hline Decade & Co-publications & Share & Single & Total \\
\hline $1955-1965$ & 4 & 28.6 & 10 & 14 \\
$1965-1975$ & 12 & 4.3 & 264 & 276 \\
$1975-1985$ & 10 & 1.7 & 569 & 579 \\
$1985-1995$ & 16 & 2.9 & 540 & 556 \\
$1995-2005$ & 14 & 16.1 & 73 & 87 \\
$2005-2017$ & 6 & 31.6 & 13 & 19 \\
Total & 62 & 4.0 & 1469 & 1531 \\
\hline
\end{tabular}


generally low. Nevertheless, also these papers, many of them collected and reprinted in his Essays of an Information Scientist, proved influential and highly cited. Moreover, through the interdisciplinarity of the field, the relatively small number of co-authors established a large collaboration network covering practically all areas of research, as we will see later on in the second-order network to be discussed in the following section. First we will have a look at his immediate collaborators, their affiliation and their research fields.

Figure 2 plots Eugene Garfield's co-authors in an ego-centric network. Many of the scientists in the ego network have only collaborated once. The size of the circles visualises the number of co-publications with Garfield. Only Sher, Pudovkin, Révész and Willjamsdorof have co-authored more frequently. The co-authors have affiliations in nine countries, namely the USA, Russia, UK, Germany, Belgium, Hungary, the Netherlands, Australia and Italy, where collaboration with Belgium and Hungary was established through the double-affiliation of one of Garfield's co-authors.

Figure 3 finally shows the institutional affiliations of Garfield's co-authors. Most institutes are located in the US and the largest number of institutional collaboration was intramural (ISI), which is, of course, in line with our expectations.

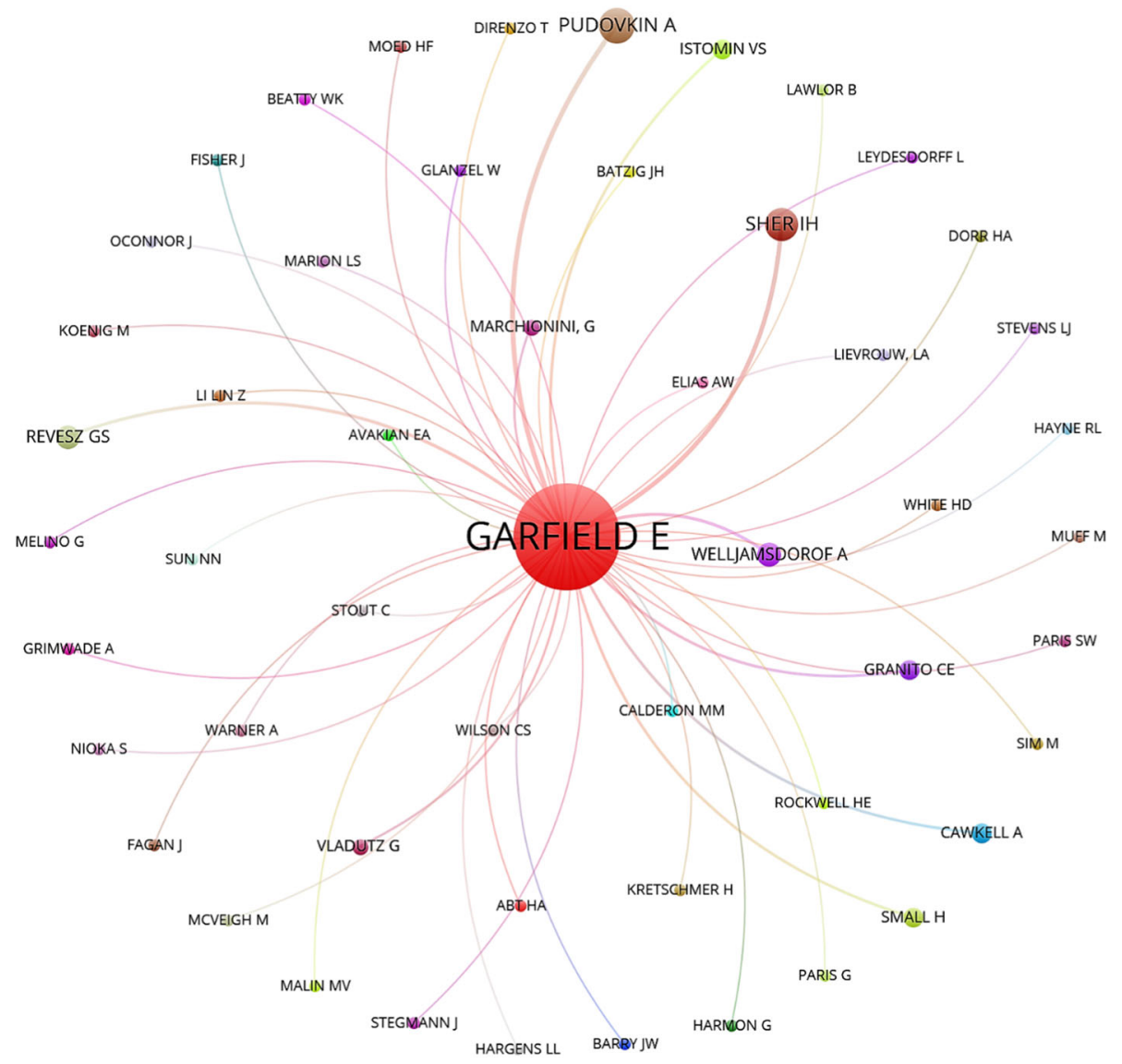

Fig. 2 Ego-network of individual co-authors of Eugene Garfield. Data sourced from Clarivate Analytics Web of Science Core Collection; visualisation: VOS Viewer 


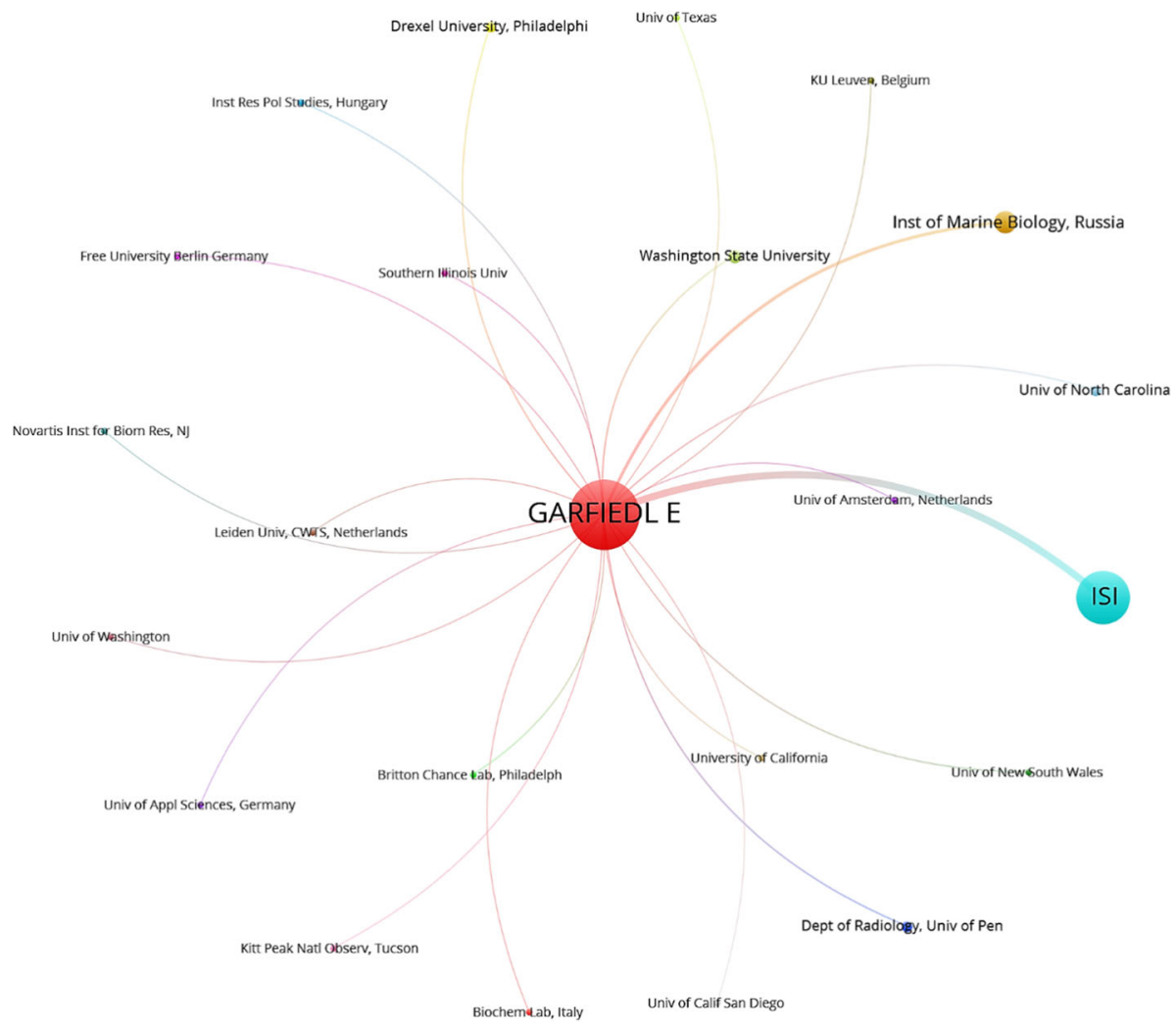

Fig. 3 Institutional affiliations of individual co-authors of Eugene Garfield. Data sourced from Clarivate Analytics Web of Science Core Collection; visualisation: VOS Viewer

\section{The second-order network (Garfield number $=2$ )}

A look at the second-order network reveals completely different patterns. In order to keep track of the readability of links and paths, we focus on one of the most central nodes in the first-order and second-order network just as pars pro toto. In the west and west-southwest of the graph in Fig. 1 we find Gerry Melino (University of Rome Tor Vergata, Italy, later also University of Leicester, UK) — Cancer Research and Molecular Biology, Shoko Nioka and Nannan Sun (both University of Pennsylvania) - Biochemistry and Biophysics. More towards north we also find Gary Marchionini (University of North Carolina) - Information Science, Jody Fagan (South Illinois University)_Library and information science, Vladimir Istomin (Washington State University)—Library and information science, Wolfgang Glänzel (KU Leuven)_mathematics and Library and information sciences, Jack Fisher (University of California San Diego)—Surgery, and in the south Helmut Abt (Kitt Peak Observatory)-Astrophysics. Zooming in on Shoko Nioka reveals the second-order network established by Nioka's co-publications (centre and bottom of Fig. 4).

As the results presented in the top network detail in Fig. 4, not all co-authors of Eugene Garfield are information scientists, nonetheless information and library science remains predominant. The activity and publication profile of the second-order networks goes far beyond the scope of information science. Here we have included all 1350 co-authors of 


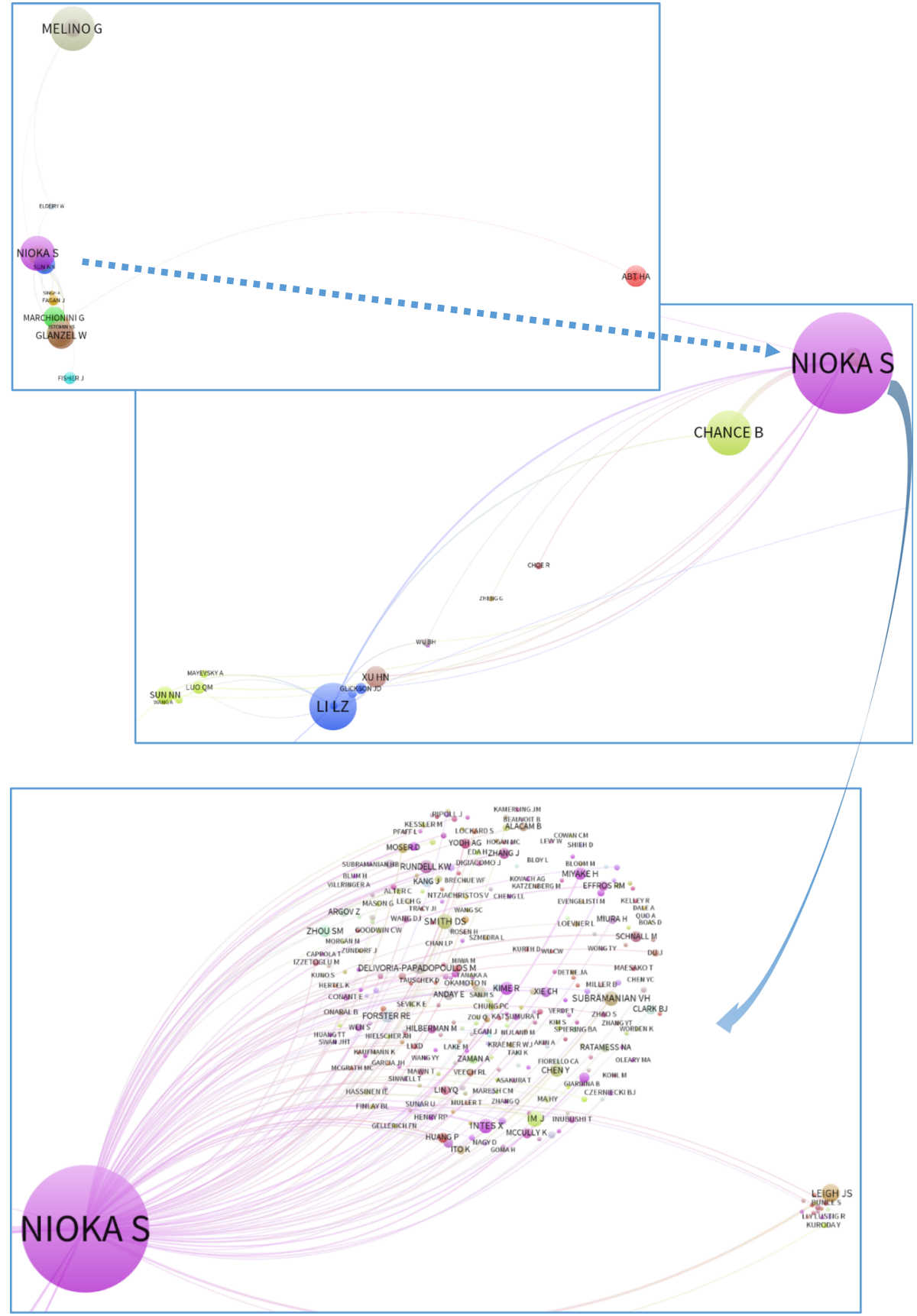

Fig. 4 Some details of the second-order co-authorship network represented by Shoko Nioka. Data sourced from Clarivate Analytics Web of Science Core Collection; visualisation: VOS Viewer 
Garfield's co-authors, that is, all scientists with Garfield number 2. We have assigned their publications to sub-fields according to the Leuven-Budapest classification scheme (Glänzel et al. 2016). The hierarchically organised classification scheme comprises 74 individual subfields (disciplines), built upon the about 250 WoS Subject Categories. The results are presented in Fig. 5. Almost all of the disciplines are covered, of course, to a different extent. As expected, information and computer science are the most important ones. The size of the circles stands for the number of papers. The large number of papers in the life sciences (followed by physics and chemistry and the others, including social sciences and humanities) testifies the large interest the corresponding scientific communities in information science and scientometrics and its impact on other research fields.

\section{Garfield's impact through co-authorship networks}

In order to underpin the last statement in the previous section, we will have a look at the citation impact within Garfield's first-order collaboration network. The first observation is already striking: At the beginning of October 2017, we observed the enormous h-index of 156 for Eugene Garfield according to the WoS. His altogether 1531 papers attracted 10,295 citations (i.e., 6.72 citations on an average) whereof the 1469 single-authored papers

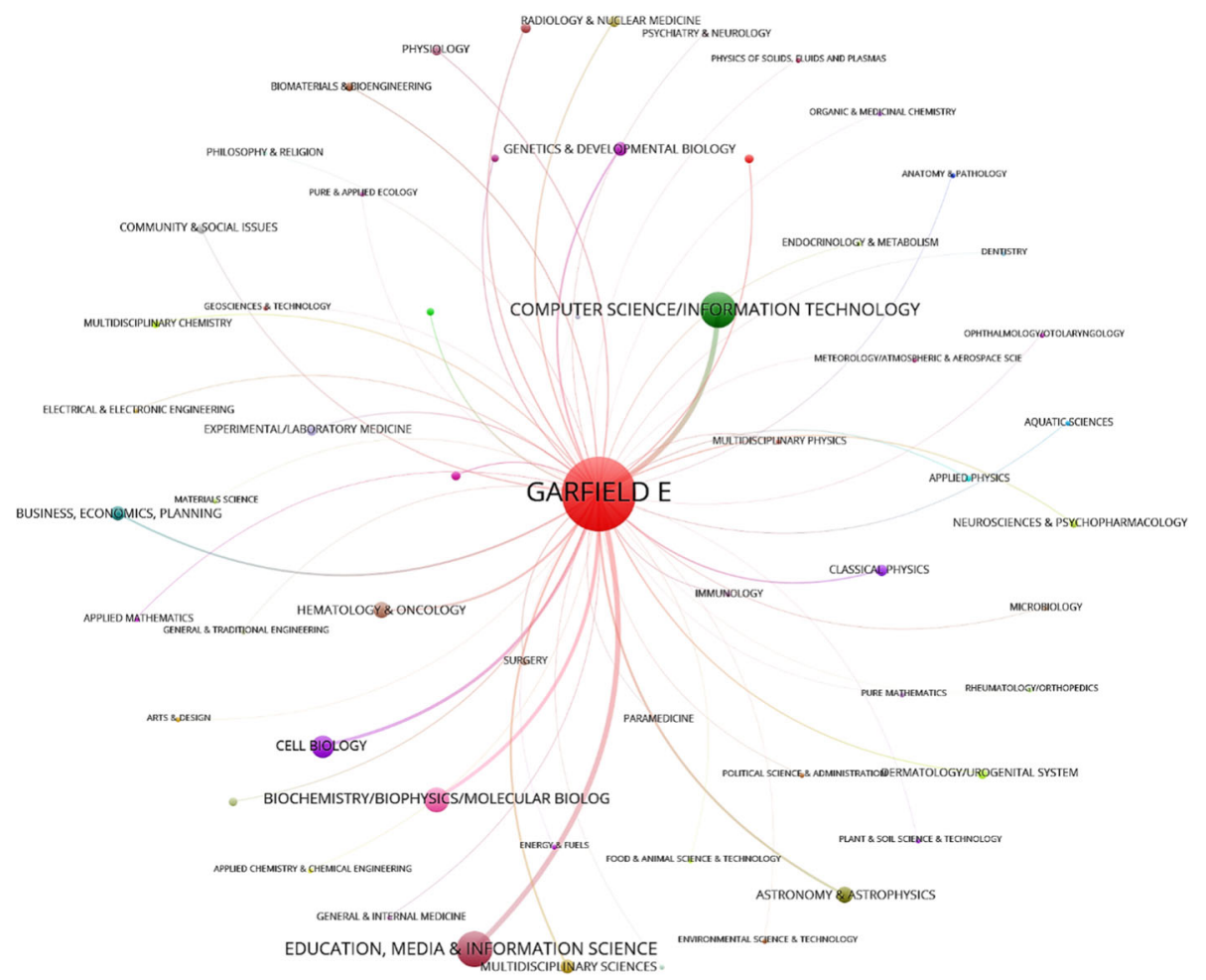

Fig. 5 Disciplines linked to Eugene Garfield through the second-order collaboration network according to the Leuven-Budapest classification scheme. Data sourced from Clarivate Analytics Web of Science Core Collection; visualisation: VOS Viewer 
received 9169 citations with a mean citation rate of 6.24 . The h-index remains unchanged if we remove co-authored papers. This is astonishing since we have not removed neither meeting abstracts nor editorials. The 564 editorials are still responsible for an h-index of 126 (with 2545 citations). The increase of citation impact of his 62 co-authored papers (1126 citations, that is, 18.16 on an average) is also in line with the expectations since here we find more research articles. It is interesting to see that the subject profile of both types of publications (single- and co-authored) are not so different as Table 4 substantiates and also the achieved citation impact by research areas (Table 5) does not substantially change either. Only the role of the two core fields, information and computer science, becomes more pronounced whenever a co-author joined the work.

The last table gives the origin of citations by countries for both single- and co-authored papers. Here we do observe some noticeable changes, namely the decrease of citations from the US for co-authored papers, which is contrasted by the increase of citations most notably from the Netherlands and Spain (see Table 6). Nevertheless, we see a word-wide reception of both types of papers with a clear dominance of the US.

\section{Conclusion and discussion}

Before we looked at the first- and second-order collaboration network generated by coauthorship links with Eugene Garfield, we had reviewed the classical case, the Erdös network. Although we used practically the same approach, we were faced with substantially different situations concerning the two networks. This is due to some fundamental issues. The Erdös number is determined on the basis of a co-publication network in mathematics using the Mathematical Reviews databases. Most vertices are representing mathematicians, so that the subject area limits the even sizeable networks to a quite neat scope. The network grows quickly up to almost to 100,000 vertices with increasing order, the scope of the database sets bounds on the growth of vertices and most notably on degrees. The second peculiarity is Paul Erdös' exceptional role in scholarly communication in mathematics. He was both exceptionally productive and co-operative in a field that was in his day, otherwise, the domain of single-authorship (cf. Glänzel 2002). By contrast, Eugene Garfield was active in an interdisciplinary field and his papers are indexed in a large multi-disciplinary database with the potential of network expansion to the complete

Table 4 Publication profile of Garfield's single- and co-authored papers by WoS Research areas. Data sourced from Clarivate Analytics Web of Science Core Collection

\begin{tabular}{lllll}
\hline Single-authored papers & & & Co-authored papers & Share $(\%)$ \\
\cline { 5 - 6 } \cline { 5 - 6 } Research area & Share $(\%)$ & & Research area & 40.3 \\
Science technology other topics & 90.3 & & Information science library science & 40.7 \\
Social sciences other topics & 78.3 & & Computer science & 38.7 \\
Information science library science & 13.3 & & Science technology other topics & 21.0 \\
Computer science & 3.5 & & Social sciences other topics & 11.3 \\
General internal medicine & 1.6 & & Chemistry & 8.1 \\
Chemistry & 1.3 & & General internal medicine & 6.5 \\
\hline
\end{tabular}


Table 5 Citation profile of Garfield's single- and co-authored papers by WoS Research areas. Data sourced from Clarivate Analytics Web of Science Core Collection

\begin{tabular}{lcclcc}
\hline Single-authored papers & & & Co-authored papers & \\
\cline { 1 - 2 } \cline { 5 - 6 } Research area & Share $(\%)$ & & Research area & Share (\%) \\
\hline Information science library science & 34.8 & & Information science library science & 54.5 \\
Computer science & 29.5 & & Computer science & 49.9 \\
Science technology other topics & 19.7 & & Science technology other topics & 10.8 \\
Social sciences other topics & 16.1 & & Social sciences other topics & 7.2 \\
Business economics & 5.3 & & Business economics & 4.8 \\
General internal medicine & 4.9 & & Engineering & 2.9 \\
Engineering & 3.5 & & General internal medicine & 2.7 \\
Psychology & 3.2 & & Chemistry & & 2.7 \\
Education educational research & 2.6 & & Education educational research & 1.8 \\
Surgery & 2.2 & & Environmental sciences ecology & 1.3 \\
\hline
\end{tabular}

Table 6 Citation profile of Garfield's single- and co-authored papers by countries. Data sourced from Clarivate Analytics Web of Science Core Collection

\begin{tabular}{|c|c|c|c|c|c|}
\hline \multicolumn{3}{|c|}{ Single-authored papers } & \multicolumn{3}{|c|}{ Co-authored papers } \\
\hline Country & Papers & Share $(\%)$ & Country & Papers & Share $(\%)$ \\
\hline USA & 2362 & 41.6 & USA & 323 & 32.2 \\
\hline UK & 416 & 7.3 & Netherlands & 95 & 9.5 \\
\hline Peoples R China & 370 & 6.5 & Spain & 88 & 8.8 \\
\hline Spain & 334 & 5.9 & Peoples R China & 82 & 8.2 \\
\hline Germany & 264 & 4.6 & UK & 70 & 7.0 \\
\hline Canada & 253 & 4.5 & Germany & 49 & 4.9 \\
\hline Australia & 227 & 4.0 & Canada & 39 & 3.9 \\
\hline Netherlands & 207 & 3.6 & Belgium & 35 & 3.5 \\
\hline Italy & 167 & 2.9 & Australia & 30 & 3.0 \\
\hline France & 150 & 2.6 & Taiwan & 25 & 2.5 \\
\hline Taiwan & 140 & 2.5 & Sweden & 24 & 2.4 \\
\hline India & 128 & 2.3 & Italy & 24 & 2.4 \\
\hline Brazil & 104 & 1.8 & Hungary & 23 & 2.3 \\
\hline Switzerland & 100 & 1.8 & India & 20 & 2.0 \\
\hline Belgium & 80 & 1.4 & Russia & 18 & 1.8 \\
\hline Japan & 74 & 1.3 & France & 18 & 1.8 \\
\hline Denmark & 73 & 1.3 & Denmark & 17 & 1.7 \\
\hline Greece & 61 & 1.1 & Switzerland & 15 & 1.5 \\
\hline Sweden & 59 & 1.0 & Brazil & 14 & 1.4 \\
\hline Hungary & 59 & 1.0 & South Korea & 12 & 1.2 \\
\hline Israel & 55 & 1.0 & Japan & 11 & 1.1 \\
\hline
\end{tabular}


subject coverage of the database. Representatives of many communities could and did enter the higher-order networks. Already the second-order network represents almost the complete coverage of the database. Like Erdős, Garfield too, was a very prolific author, yet he was by far not so co-operative although information science is not really a domain of single-authorship. The different communication patterns of two prominent researcher personalities along with the peculiarities of their research fields result in different patterns. The Garfield network allows interesting insight into a universe that is less "bounded" than the mathematics network and into the dissemination of ideas by forming co-authorship based communities.

\section{References}

Braun, T., Glänzel, W., \& Schubert, A. (2001). Publication and co-operation patterns of the authors of neuroscience journals. Scientometrics, 51(3), 499-510.

Braun, T., Glänzel, W., \& Schubert, A. (2017). Obituary. Eugene Garfield (1925-2017). Scientometrics, $111(1), 1$.

Erdős Number Project. (2017). Oakland University. https://oakland.edu/enp/. Data retrieved in August 2017.

Glänzel, W. (2002). Co-authorship patterns and trends in the sciences (1980-1998). A bibliometric study with implications for database indexing and search strategies. Library Trends, 50(3), 461-473.

Glänzel, W. (2017). Eugene Garfield (1925-2017). ISSI Newsletter, 13(1), 1-2.

Glänzel, W., Thijs, B., \& Chi, P. S. (2016). The challenges to expand bibliometric studies from periodical literature to monographic literature with a new data source: The Book citation Index. Scientometrics, 109(3), 2165-2179.

Goffman, C. (1969). And what is your Erdős number? American Mathematical Monthly, 76(7), 791. 